\title{
Hábitos de consumo de alcohol en población universitaria
}

\author{
Beatriz CABREJAS MartíneZ \\ cmbea79@usal.es \\ Ángeles LlorCA DiEZ \\ tanamanca@hotmail.com \\ Teresa Gallego Álvarez \\ gallego.tr@gmail.com \\ Gloria Bueno CARRERA \\ Universidad de Salamanca \\ gloriabueno@usal.es \\ Ma Ángeles Diez SÁNCHEZ \\ Universidad de Salamanca \\ madiez@usal.es
}

\section{Resumen:}

El presente trabajo tiene como objetivo realizar una investigación sobre los hábitos de consumo de alcohol, en un estrato de la sociedad muy concreto como es el de estudiantes universitarios, concretamente de Salamanca; en una franja de edad y condicionantes sociales que aumentan la vulnerabilidad que presenta este grupo de población; y en una ciudad que tiene fama de tener un importante ambiente nocturno, gracias a los más treinta mil estudiantes que pasan cada año por ella.

Nuestros resultados indican que, el perfil del estudiante universitario consumidor de alcohol es varón, que se inicia en el consumo aproximadamente a los 14 años y que con 16 tiene su primera intoxicación etílica. En la actualidad se embriaga una vez al mes en compañía de sus amigos, bebe principalmente combinados, y no mezcla con otras drogas, y todo ello, bajo un gran desconocimiento por parte de sus padres.

Palabras clave: Consumo de alcohol; Patrón de Consumo; Cambio social; Universitarios

\section{Alcohol drinking habits in college population}

\begin{abstract}
:
The present paper aims to conduct a research on the alcohol consumption habits, in a very specific stratum of society such as university students, specifically of Salamanca. They are in an age and social conditions that increase their vulnerability, and they live in a city that is reputed to have an important nightlife, because of the over thirty thousand students who pass through it per year.

Our results show that the profile of the college student alcohol consumer is a male, which begins on his consumption when he is 14 years old and on his 16 years old has his first alcohol intoxication. Currently, he gets drunk once a month in the company of his friends, he drinks mainly mixed drinks and he does not mix it with other drugs, doing all of this under a wide ignorance of his parents.
\end{abstract}

Key Words: Alcohol consumption; Consumption pattern; Social change; University Students 


\section{Referencia normalizada:}

Cabrejas Martínez, B., Llorca Diez, A., Gallego Álvarez, T., Bueno Carrera, G. y Diez Sánchez, M. A. (2014): Hábitos de consumo de alcohol en población universitaria. Historia y Comunicación Social. Vol. 19. Núm. Especial Marzo. Págs. 775-789.

Sumario: 1. Introducción, 2. Estado actual de la cuestión, 3. Metodología, 4. Muestra, 5. Resultados, 6. Conclusión, 7. Bibliografía

\section{Introducción}

A lo largo de la historia, las diversas sociedades humanas han conocido y utilizado diferentes productos para inducir alteraciones en las funciones psíquicas. En muchas ocasiones el consumo de sustancias se realizaba en el marco de actividades socializadoras y muy estructuradas, o dentro de rituales de iniciación o adhesión a una comunidad (Casas Brugué, Roncero Alonso y Colom Farran, 2005). Sin embargo, en cada una de las diversas situaciones, el objetivo de su consumo ha ido encaminado a sedar y paliar el dolor o bien a estimular y experimentar sensaciones placenteras, o para relacionarse.

Entre todas estas sustancias se encuentra el alcohol, ya disponible desde la época neolítica, cuando la invención de la cerámica hizo posible el almacenamiento de frutos, vegetales, miel, y otros productos ricos en hidratos de carbono que con su fermentación generaban alcohol (Santo-Domingo, Gual y Rubio, 2005).

Prácticamente todas las culturas nos han dejado testimonios escritos sobre el alcohol y sus efectos, y se remontan a poco después de haberse desarrollado la escritura, lo que indica que ya entonces tenían una amplia experiencia sobre dicha sustancia (Ladero y Lizasoain, 2009). Es de hecho, la primera droga a la que los textos históricos se han referido en términos de abuso, varios miles de años antes de Cristo (Blum, 1973). El libro del Génesis hace referencia en el pasaje de Noé: (Gén 9, 18-27) (cit. Por Díaz, 2001), afirmando que se dedicó a la agricultura y plantó una viña pero cuando bebió vino, se embriagó y quedó tendido en medio de su tienda, completamente desnudo.

El profeta Isaías describe el día después de una borrachera, que interpretamos como el periodo de resaca, lamentándose de aquellos que madrugan para correr tras la bebida y hasta muy entrada la noche se acaloran con el vino. Posteriormente, Homero relata en la Odisea los efectos del alcohol y el fatal desenlace que sufrió el joven remero llamado Elpenor, el cual, después de una noche de consumo excesivo de vino, también en plena resaca, tropezó, sufrió una caída desde un tejado y se partió el cuello (Díaz, 2001).

El alcohol ha sido sin duda la droga por excelencia de los pueblos mediterráneos. Sus efectos embriagantes fueron utilizados como vínculo litúrgico por egipcios, griegos, romanos y hebreos. La vid se ha considerado como símbolo de la vida y de la inmortalidad. Así, los sumerios (habitantes de Mesopotamia hace tres mil años) 
representaban la vida con el signo de una hoja de parra y utilizaban bebidas fermentadas, citadas incluso en el Código de Hammurabi, donde se prescribía la pena de ser arrojado al río para aquellos propietarios de locales de bebidas que permitieran la embriaguez de sus clientes (Pons Diez y Berjano Peirats, 1999; Díaz, 2001).

Fueron los egipcios quienes inventaron la cerveza unos tres mil años antes de Cristo, vinculando su consumo a actividades populares, elaboraron normas y advertencias sobre el uso y el abuso de dicha sustancia y la gravaron con algo similar a lo que ahora llamaríamos impuestos. Asimismo, utilizaron el vino hasta bien avanzada la dominación romana, como vínculo de unión con la espiritualidad, donde lo consumían para la embriaguez sagrada en el templo de Tentyra, dentro del culto dedicado a Hator, diosa egipcia del amor (Pons Diez y Berjano Peirats, 1999).

El culto que las antiguas civilizaciones griega y romana ofrecían a sus respectivos dioses del vino -Dioniso y Baco- es otro excelente indicador del arraigo que esta bebida ha tenido en los cultos religiosos de los pueblos mediterráneos. El vino era el sustituto de la sangre del dios Dioniso, y con el objetivo de alcanzar la inmortalidad, se celebraban rituales en los que se incluían procesiones y danzas, buscando los participantes la unión con el dios a través de un estado de embriaguez. Esta ceremonia fue recogida por la cultura latina, adoptando el nombre de Baco y el ritual festivo-religioso conocido como bacanal, ha llegado hasta nuestros días como ejemplo de manifestación lúdica y liberadora de instintos, mediante la utilización de un producto divinizado: el vino (Diaz, 2001; Pons Diez y Berjano Peirats, 1999).

Por su parte, el sincretismo judeo-cristiano mantuvo esta sustancia como vehículo de encarnación de la divinidad. Es más, para las culturas mediterráneas de la antigüedad el vino era la "sangre de la tierra", de ahí que adquiera unas cualidades mágicas que persisten en la liturgia cristiana (Oberlé, 1989). La expansión del Imperio Romano contribuyó a la difusión de la vid por todos los territorios dominados, siendo los primeros en promulgar leyes sobre su cultivo y comercio (Sournia, 1990). De tal manera que, después de la caída del imperio romano existen indicios, que entre los musulmanes que habitaban los reinos peninsulares, alguna selecta minoría era consumidora de vino (Freixa, 1993).

A partir del siglo XIV el alcohol fue una sustancia importante que se usaba, además de cómo medicamento revitalizador, cómo analgésico y euforizante, relacionado en particular con la epidemia de la peste negra (1347-1351), como disolvente en la preparación de perfumes y otros productos variados, en relación con usos religiosos $\mathrm{y}$ en el trueque por otros menesteres, expandiéndose por el norte y este de Europa, donde su uso adquirió patrones de consumo diferentes de los mediterráneos preexistentes (Santo-Domingo, Gual y Rubio, 2005).

Así, en diversas regiones de Europa y casi siempre en los monasterios, se van elaborando los primeros licores destilados que alcanzan fama y relevancia: cognac, benedictine, chartreuse, llegando a Irlanda y Escocia, donde en este último país, en el siglo XV comienza a elaborarse un aguardiente de cebada llamado visge beatha (agua de la vida en gaélico escocés) constituyendo el primer antecedente del whisky 
(Pons Diez y Berjano Peirats, 1999). Al mismo tiempo, entre los siglos XIII y XV, en la abadía benedictina de Sankt Gallen, en Suiza, comienza a desarrollarse una industria cervecera, con una elaboración de un producto semejante al actual, que no llega a la península hasta el siglo XVI, con el emperador Carlos V (1500-1558). Sin embargo, no es aceptado por el pueblo al considerarlo una bebida extranjera. De hecho, el uso popular de la cerveza en España no llegará hasta mediados del siglo XIX, cuando industriales alemanes ubican sus factorías cerveceras en Cataluña (Pons Diez y Berjano Peirats, 1999).

En el siglo XVII, comerciantes y propietarios agrarios españoles y portugueses inician la exportación y plantación masiva de caña de azúcar en las Antillas y Brasil, con lo que se desarrolló la producción y difusión del ron. Por otro lado, en Francia, el monje benedictino dom Pierre Pérignon (1638-1715) da lugar a lo que hoy conocemos como champán. Junto a esto, la investigación científica del químico francés Louis Pasteur (1822-1895) permitió un mayor conocimiento de los procesos de fermentación y destilación, lo que posibilitó la industrialización y producción de alcohol, así como su perfección y abaratamiento. De esta manera, se comenzó con la mercantilización moderna del vino y de otras bebidas alcohólicas, y a continuación sucedió lo mismo con los destilados (Santo-Domingo, Gual y Rubio, 2005).

La mayor y mejor oferta de alcohol dio lugar a una disponibilidad más fácil, lo que unido a los cambios sociales, económicos y culturales fruto de la nueva sociedad industrial, contribuye a un incremento en el consumo de bebidas alcohólicas (Pons Diez y Berjano Peirats, 1999). En consecuencia, los primeros problemas sanitarios y sociales graves relacionados con el consumo de alcohol por capas amplias de la población se dieron en Inglaterra, en el siglo XVIII, con la popularización de la ginebra (Ladero y Lizasoain, 2009). Posteriormente, su inclusión como sustancia "estimulante" de moda entre la llamada bohemia artística y literaria de Europa y América del Norte (Toulouse-Lautrec, Baudelaire, Poe,...), buscando sus efectos embriagantes, desinhibidores y ansiolíticos, alcanzaron gran auge entre la población occidental del siglo XIX, donde se habla del alcoholismo como un problema social y sociosanitario de primera magnitud (Santo Domingo, 1990).

Curiosamente, es a partir de este momento cuando desde diversas instancias de la Iglesia Católica y Protestante se inician campañas redentoras de este mal social. La elevación drástica de los impuestos sobre las bebidas alcohólicas, la limitación de los lugares de consumo y el rigor en el castigo de la destilación y el comercio ilegal consiguieron amainar considerablemente el problema (Ladero y Lizasoain, 2009).

Asimismo, entre 1920 y 1933 en los Estados Unidos, se extiende la Ley Seca, que según Martinez-Azumendi (2009) es el resultado del movimiento prohibicionista de los grupos religiosos protestantes, apoyados por sectores anti-germánicos de la Primera Guerra Mundial y las mujeres del movimiento sufragista. Todo ello, desencadenó la prohibición de la elaboración, venta o transporte de licores intoxicantes (cualquier bebida con más de un $0,5 \%$ de graduación alcohólica), que derivó en la reducción del consumo, pero al mismo tiempo incrementó y desarrolló el crimen 
organizado, con destilerías clandestinas controladas por gángsters. Igualmente, en diferentes periodos del siglo XX también se promulgaron leyes de prohibición en Chile, Rusia, Islandia, Noruega, Finlandia o diversos estados de la India (Rodríguez García, 2010).

Sin embargo, durante el siglo XX se produjo una globalización de los patrones de uso; hasta la primera mitad, cada país tenía unos hábitos de consumo propios, basados en sus bebidas autóctonas: el área mediterránea se caracterizaba por la utilización de vino, preferentemente asociado a las costumbres de alimentación, progresivamente estos patrones se han ido sustituyendo por las prácticas de los países anglosajones.

En 1849 Magnus Huss introduce por primera vez el término "alcoholismo". Hasta ese momento este síndrome era entendido como una dipsomanía, es decir, un problema secundario originado por anomalías mentales que era transmitido de padres a hijos, produciendo en las generaciones una mayor degradación física y moral. Por ello, se proponía un tratamiento con una importante carga moralizante. Así, a finales del siglo XIX y principios del XX se observa que el consumo excesivo de alcohol produce determinadas lesiones que causan síntomas biológicos y conductuales, existencia de una tendencia irresistible a beber y la idea de que algunas alteraciones del Sistema Nervioso Central son la causa de la enfermedad (Santo- Domingo, Martínez y Rubio, 1997).

\title{
2. Estado actual de la cuestión
}

Los problemas relacionados con el consumo de alcohol en las personas jóvenes tienden a aumentar, convirtiéndose en patológico cuando comienzan a depender psicológicamente del alcohol, es decir, cuando lo necesitan de forma impulsiva para buscar estimulación, ocultar la propia inseguridad personal o compensar la falta de expectativas de futuro. Por ello, se puede hablar de consumo abusivo por parte de los adolescentes cuando el alcohol interfiere negativamente en el funcionamiento psicológico, en el rendimiento académico o laboral, en las relaciones sociales y en la vida familiar. En estos casos la vida del adolescente comienza a girar en torno al alcohol, como forma de divertirse, de relajarse, de mostrarse agresivo, etc., y todo lo demás pasa a ser secundario (Tabla 1)

Tabla 1. Indicadores de riesgo de los jóvenes en rehación con el akoholismo en ba vida adulta (Echeburúa, 2001)

\author{
$>$ Consumo excesivo de alcohol, con borracheras frecuentes \\ $>$ Utilización de otras drogas \\ $>$ Conductas pendencieras y antisociales \\ $>$ Dificultad deadaptaciónescolar o laboral \\ $>$ Accidentabilidad \\ $>$ Inestabilidad emocional y búsquedacontinua de estimnlación \\ $>$ Faltadeaficiones e intereses \\ DProblemas en las relaciones interpersonales.Amigos consumidores dealcohol \\ $>$ Relaciones sexuales sin protección \\ $>$ Lazos familiares débiles. Consumo dealcohol abusivo en la familia
}

His

Vol. 19.No Esp.Marzo (2014) 777-789 
Las pautas de consumo entre los jóvenes aquejados de una ingesta excesiva de alcohol presentan unas características distintivas de las de los adultos (Tabla 2).

Tabla 2. Diferencias entre adolescentes y adultos con problemas de abuso de alcohol (Harford, Fadery Chen citado por Parada, 2009)

\begin{tabular}{|c|c|}
\hline Adolescentes & Adultos \\
\hline $\begin{array}{l}\text { > Tendencia al patrón de consumo binge } \\
\text { drinking. } \\
\text { >Corta historia de consumo (entre uno y } \\
\text { dos años). } \\
\text { >Más problemas relacionados con el } \\
\text { consumo dealcohol (familiares, } \\
\text { académicos y/o laborales) } \\
\text { DAlta prevalencia de dos síntomas de } \\
\text { dependencia en los que se suele basar el } \\
\text { diagnóstico: tolerancia al alcohol y } \\
\text { consumo de alcohol en mayores } \\
\text { cantidades de lo que en un principio } \\
\text { pretenden. } \\
\text { Enalgnnos casos, presencia desíntomas } \\
\text { leves de abstinencia }\end{array}$ & $\begin{array}{l}\text { Patróndeconsumo regular. } \\
\text { PHistoria de consumo de al menos } 5 \\
\text { años. } \\
\text { >Menos problemas relacionados con el } \\
\text { consumo dealcohol (familiares y/o } \\
\text { laborales). } \\
\text { Pstablecimiento de dependencia basado } \\
\text { principalmente en la adicción física (como } \\
\text { el síndromedeabstinencia). }\end{array}$ \\
\hline
\end{tabular}

Los problemas de dependencia física o los daños somáticos son, habitualmente escasos. En concreto, el exceso de alcohol en estas edades se caracteriza por ser de una menor frecuencia pero de mayor intensidad (Reboussin, Ip y Wolfson, 2008), lo que suele estar asociado a borracheras frecuentes y embriagueces atípicas (Vasallo, Jáimez y Rubio, 2002). Esta pauta de consumo juvenil condiciona de manera muy importante la formación académica, generando un mal rendimiento escolar (Llorca, et al., 1995), lo que se asocia a un bajo interés por los estudios, no asistencia a clase, poca motivación, y baja autoestima, etc. (Piko y Kovács, 2010), que repercute en los futuros estilos de vida y las posibilidades laborales. De hecho, las estadísticas muestran que estos jóvenes presentan un mayor número de conductas de riesgo (Calafat, Blay, et al., 2009) pudiendo: sufrir y morir en accidente de tráfico antes de los 25 años (DGPNSD, 2007); verse involucrado en actos antisociales que deriven en consecuencias legales, cárcel y marginación (Contreras Martínez, Molina Banqueri y Cano Lozano, 2012); e implicación en relaciones sexuales no deseadas, al convertirse en actores o víctimas de una agresión sexual (Fossos, et al., 2011); o encuentros sexuales sin protección, no teniendo en cuenta los riesgos de las mismas (embarazos no deseados, enfermedades de transmisión sexual, sida, etc.) (Griffin, Umstattd, et al., 2010).

Por tanto, la mayor propensión de los adolescentes y jóvenes hacia estos patrones de consumo intensivos (binge drinking) e intermitentes, podría tener importantes efectos en el rendimiento neuropsicológico, similares a los déficits observados en bebedores crónicos (Scaife y Duka, 2009). Estas funciones, además, estarían muy 
probablemente moduladas a nivel individual por otros factores como riesgo genético, sexo, edad de inicio o policonsumo (Cadaveira, 2009).

En este contexto, parece elemental, estudiar el tipo de motivaciones asociadas al consumo de alcohol. La motivación es definida como aquella necesidad o deseo que sirve para activar la conducta y orientarla hacia una meta (Myers, 1999). En este sentido, dentro de las motivaciones que los adolescentes consideran relevantes para iniciar su consumo de alcohol existen diferencias entre autores. Por un lado, López et al. (2001) afirman que el motivo de beber por primera vez es en la mayoría de los casos debido a una fiesta o celebración, seguidos de la curiosidad, el ofrecimiento de los padres y por no quedar mal ante los compañeros. Es decir, el inicio del consumo parece estar relacionado con variables como la presión del grupo y la afirmación de la propia identidad, dicho de otra forma, utilizan el alcohol como un facilitador de las relaciones sociales, un elemento de aceptación, aprobación y cohesión entre el grupo de iguales que es fundamental para la diversión, más que con la curiosidad o la novedad del consumo. Por tanto, los primeros consumos y los que le siguen suelen ser un verdadero acto grupal, en el que el adolescente necesita del grupo para que le enseñe a consumir y para que de sentido a su consumo, haciendo lo que los demás esperan que haga (Giró, 2007; De la Villa Moral y Ovejero, 2011).

Por otra parte, también existen motivaciones para no beber, entre las que destacan aquellas que tienen que ver con los efectos molestos de la borrachera, con las consecuencias negativas sobre la salud, por no querer perder el control y porque consideran desagradable o no les gusta el sabor. Estos motivos son seguidos por la posibilidad de provocar accidentes, el riesgo de una adicción al alcohol, hacer el ridículo, las reprimendas de la familia, la posibilidad de ser castigados o sancionados, la falta de dinero y el tener relaciones sexuales de riesgo (Giró, 2007) (Tabla 3).

Tabla 3. Motives para bebery no beber (Encuesta escolar del Plan Nacional sobreDrogas, 2002)

\begin{tabular}{|c|c|}
\hline Motivos para beber & Motivos para no beher \\
\hline$>$ Les gusta el sabor (73\%) & $>$ Efectos negativos para la sahud $(58,5 \%)$ \\
\hline $\begin{array}{l}\text { Diversión y placer }(58,5 \%) \\
\text { > Olvidar problemas personales (15\%) }\end{array}$ & $\begin{array}{l}\text { PPérdida decontrol y efectos desagradables } \\
(45,5 \%)\end{array}$ \\
\hline $\begin{array}{l}\text { Sentir emociones nuevas }(12,9 \%) \\
\text { S Superarla timidez, relacionarse }(11,2 \%)\end{array}$ & $\begin{array}{l}\text { Piesgo de provocaraccidentes }(33,3 \%) \\
\text { Efectos molestos }(30,9 \%)\end{array}$ \\
\hline
\end{tabular}

Todo ello genera un aumento de los consumos problemáticos y de los porcentajes de bebedores de riesgo con incrementos de urgencias hospitalarias relacionadas con el uso toxicofílico del alcohol (Observatorio Español sobre Drogas, 2002, 2004), al igual que la experimentación con otras sustancias psicoactivas, tales como derivados cannábicos (Olivar y Carrero, 2007) y psicoestimulantes (Mantecón, 2008) asociados a la cultura lúdica y recreativa juvenil (De la Villa Moral y Ovejero, 2011). 
Quizás estos hechos hayan provocado la campaña que desde el Plan Nacional sobre Drogas, presentaba el Delegado D. Francisco Babín y que se ha llamado "Los que no". La noticia era difundida a través de la agencia EFE el 5 de junio. Se instaba, a estar alerta sobre las graves consecuencias del inicio temprano del alcohol. En la misma rueda de prensa, afirmó que el Gobierno estaba estudiando sancionar con multas a los padres de los menores que acuden a las urgencias hospitalarias en situaciones de intoxicación y coma etílico, ya que esta conducta se entiende como una dejación de la tutela efectiva por parte de los progenitores.

\section{Metodología}

Por todo ello, el presente trabajo tiene como objetivo realizar una investigación sobre los hábitos de consumo de alcohol, en un estrato de la sociedad muy concreto como es el de estudiantes universitarios, concretamente de Salamanca; en una franja de edad y condicionantes sociales que aumentan la vulnerabilidad que presenta este grupo de población; y en una ciudad que tiene fama de tener un importante ambiente nocturno, gracias a los más treinta mil estudiantes que pasan cada año por ella.

Se trata de un estudio transversal, cuya muestra fue recogida mediante un muestreo intencionado por conglomerados. Los criterios de inclusión fueron:

- Ser estudiante de la Universidad de Salamanca.

- De ambos sexos.

- Sin problemas físicos y/o psicológicos que impidiesen la realización de las pruebas.

- Participar de manera voluntaria en el estudio.

- El dossier estaba compuesto por

- Una entrevista semiestructurada donde se recogían datos personales, familiares, socioculturales y académicos de los participantes, y

- Una encuesta sobre diversas características relacionadas su consumo etílico, tanto en la forma de inicio, como en el consumo posterior.

\section{Muestra}

La muestra quedó formada por 849 sujetos, con edades comprendidas entre los 17 y 30 años. La media de edad era de 20,58 años con una desviación típica de 2,06.

El porcentaje de hombres y mujeres muestra un predominio de éstas (67\%) frente al 33\% de los varones. Resultados acordes con los proporcionados tanto por la propia 
Universidad de Salamanca (Unidad de Evaluación de Calidad, 2012) como por el Ministerio de Educación (2011), donde al igual que en la inmensa mayoría de los Países Europeos, hay un mayor porcentaje de mujeres con estudios superiores.

Los datos de nuestro estudio evidencian que el $63,1 \%$ de los universitarios manifiestan ser educados bajo un estilo democrático, seguidos del modelo permisivo $(20,4 \%)$ y autoritario $(10,8 \%)$. La relación con sus progenitores mayoritariamente la valoran como muy buena $(45,1 \%)$ y buena $(41,3 \%)$, frente a un $12,3 \%$ que creen que la relación entre sus padres no es positiva.

A lo largo del curso académico el $41 \%$ de los universitarios vive en un piso compartido, el $37 \%$ vive junto a su familia, un $15 \%$ se aloja en una residencia y un $7 \%$ en un piso sólo.

Con respecto a los estudios cursados por nuestra muestra de universitarios, observamos que el $60 \%$ se encuentra realizando alguna licenciatura, seguido de un $39,7 \%$ que cursa una diplomatura, el resto, llevan a cabo otro tipo de estudios, como son los de Tercer Ciclo o Títulos Propios, cómo es el de Criminología.

\section{Resultados}

A continuación se exponen los resultados de la encuesta aplicada a la muestra con la finalidad de conocer los hábitos de consumo de parte de una población universitaria, concretamente de Salamanca.

- La edad a la que los estudiantes de nuestra muestra admiten haber probado alcohol por primera vez oscila entre los 3 y los 22 años, aunque la media se sitúa en los 14,43 años, con una variabilidad de 2 años.

- El 83,9\% de los participantes estaban acompañados por sus amigos la primera vez que bebieron alcohol, seguidos por el $11,7 \%$ que lo probaron junto a sus padres. Dato acorde con la literatura, que manifiesta que aunque tradicionalmente el ámbito familiar, a veces era el más propicio para el inicio en el consumo de alcohol (sobre todo en ocasiones de fiesta o celebración), es en el grupo de iguales donde se favorecen las conductas consumistas de esta sustancia (Fundación Pfizer, 2012).

- Los diferentes tipos de bebida escogidas por los participantes de este estudio, para el inicio de su consumo son las siguientes (Tabla 4). 


\section{Tabla 4. Tipos de bebida de inicio}

\begin{tabular}{ll}
\hline Bebidas & Porcentaje \\
\hline Vino/champan & $21,6 \%$ \\
Cerveza/sidra & $15,8 \%$ \\
Aperitivos & $1,1 \%$ \\
Combinados & $42,8 \%$ \\
Chnpitos & $10,8 \%$ \\
Licores solos & $3,4 \%$ \\
$* *$ Varias bebidas & $1,3 \%$
\end{tabular}

- Los principales motivos para beber por primera vez fueron: saber cómo es en un $58,9 \%$; seguido de por pasarlo bien $(36,5 \%)$; por otros motivos $(8,9 \%)$; o por quedar bien con los amigos $(5,9 \%)$.

- Al preguntar sobre la experiencia de la embriaguez, en alguna ocasión a lo largo de su vida, el $83 \%$ de los estudiantes universitarios contestaban afirmativamente. La edad media de esta primera embriaguez se situó en 16,12 años, con una desviación típica de 1,92.

- Únicamente el $21 \%$ de nuestra muestra manifiesta que sus padres tuvieron conocimiento de su primera embriaguez, lo que significa que, nada menos que un $79 \%$, estuvieron ajenos, no se enteraron de la conducta arriesgada de sus hijos. Además, entre aquellos sujetos que afirman que sus padres conocieron este suceso, existen diferentes formas de reaccionar, en el 7,8\% su reacción fue neutra, seguida de valoraciones negativas $(5,5 \%)$ y muy negativas $(3,4 \%)$ y sorprendentemente un $1,3 \%$ y un $0,4 \%$, respondieron ante esta embriaguez de sus hijos de manera positiva y muy positivamente, respectivamente.

- Un $25,7 \%$ de los que se habían embriagado alguna vez no han vuelto a repetir esta experiencia; sin embargo, el $15,5 \%$ ha seguido manteniendo esta conducta los fines de semana, el 16,1\% lo ha hecho de dos a cuatro veces al mes, el 2,1\% varias veces por semana y el mayor porcentaje se encuentra entre aquellos sujetos que se han embriagado una ó menos veces al mes $(38,2 \%)$.

- Ante la pregunta, “¿Ha estado embriagado en los últimos 30 días?” el 48\% afirma que sí, son 407 sujetos. El tipo de bebida que es más frecuente en el consumo actual de los estudiantes universitarios salmantinos, son los combinados $(65,6 \%)$, seguidos de la cerveza/sidra $(38,5 \%)$, chupitos $(16,5 \%)$, vino/ champan (16\%), licores solos (10,4\%) y finalmente, aperitivos.

- El aspecto positivo de la encuesta es que tan solo un 10\% de los participantes consume otras drogas diferentes al alcohol, siendo la más frecuente el tabaco $(4,6 \%)$, seguido del cannabis $(3,5 \%)$ y el policonsumo $(0,9 \%)$; en menor medida se ingieren las anfetaminas y el pegamento (0,1\%) (Fig. 69 y 70). 


\section{Conclusión}

El perfil del estudiante universitario consumidor de alcohol es varón, que se inicia en el consumo aproximadamente a los 14 años y que con 16 tiene su primera intoxicación etílica. En la actualidad se embriaga una vez al mes en compañía de sus amigos, bebe principalmente combinados, y no mezcla con otras drogas, y todo ello, con un gran desconocimiento por parte de sus padres, tutores y familiares más directos.

\section{Bibliografía}

\subsection{Libros}

BLUM, R. H. (1973). “Un hombre de ciencia juzga la droga”. En: L. Daufí (Ed.). La verdad sobre la droga. Barcelona: Promoción Cultural.

CASAS BRUGUÉ, M., RONCERO ALONSO, C. Y COLOM FARRAN, J. (2005). "Avances en conductas adictivas". En J. Vallejo Ruiloba (Ed). Tratado de Psiquiatría. Volumen I. Barcelona: Ars Médica.

DÍAZ, F. A. (2001). Hablamos de alcoholismo. Madrid: San Pablo.

FREIXA, F. (1993). "Uso y riesgo de dependencia del alcohol en adolescentes y jóvenes". En: M. Sánchez-Turet (Ed.), Uso, abuso y dependencia del alcohol en adolescentes y jóvenes. Barcelona: PPU.

GIRÓ, J. (2007). Adolescentes, ocio y consumo de alcohol. Madrid: Entinema.

LADERO, J. M. Y LIZASOAIN, I. (2009). "Alcohol (I): farmacología del alcohol. Intoxicación aguda”. En P. Lorenzo, J. M. Ladero, J. C. Leza e I. Lizasoain (Eds.), Drogodependencias. Farmacología. Patología. Psicología. Legislación (pp. 385-400). Editorial Medica Panamericana S.A. ( $\left.3^{\mathrm{a}} \mathrm{ed}\right)$.

MYERS, D. G. (1999). Psicología. $5^{\text {a }}$ edición. Madrid: Editorial Médica Panamericana, S. A.

OBERLÉ, G. (1989). Les fastes de Bacchus et de Comus. París: Belford.

RODRÍGUEZ GARCÍA, F. D. (2010). Alcohol y cerebro. Cádiz: Ediciones Absalon, S. L.

SANTO DOMINGO, J. (1990). El alcohol. Madrid: Rialp.

SANTO-DOMINGO, J., GUAL, A., RUBIO, G. (2005). "Adicciones a sustancias químicas (I). Alcohol”. En J. Vallejo y C. Leal (Eds.). Tratado de Psiquiatría. Vol. I. (pp.750-771). Editorial: Ars Medica.

SOURNIA, J.C.H. (1990). A history of alcoholism. Londres: Basil Blackwell.

VASALLO, J. L., JÁIMEZ, M. Y RUBIO, A. (2002). "Trastornos relacionados con el consumo de drogas y alcohol”. En: Rubio Flores, A., Ortega Basanta, L. (Coord.), Manual de psiquiatría penitenciaria (pp. 47-87). Madrid. Egraf. Wyeth.

7.2 Revistas científico-profesionales

CADAVEIRA, F. (2009). “Alcohol y cerebro adolescente”. Adicciones, 21 (1), 9-14. 
CALAFAT, A., JUAN, M. Y DUCH, M. A. (2009). "Intervenciones preventivas en contextos recreativos nocturnos: revisión”. Adicciones, 21 (4), 387-414.

CONTRERAS MARTÍNEZ, L., MOLINA BANQUERI, V. Y CANO LOZANO, M C. (2012). "Consumo de drogas en adolescentes con conductas infractoras: análisis de variables psicosociales implicadas". Adicciones, 24 (1), 31-38.

DE LA VILLA MORAL, M. Y OVEJERO, A. (2011). "Consumo abusivo de alcohol en adolescentes españoles: Tendencias emergentes y percepciones de riesgo". Universitas Psichologica, 10 (1), 71-87

ECHEBURÚA, E. (2001). Abuso de alcohol. Guía práctica para el tratamiento. Madrid. Editorial Síntesis, S. A.

FOSSOS, N., KAYSEN, D. ET AL. (2011). "Coping motives as a mediator of the relationship between sexual coercion and problem drinking in college students". Addictive Behaviors, 36 (10), 1001-1007.

GRIFFIN, J. A., M. R. UMSTATTD, ET AL. (2010). "Alcohol use and high-risk sexual behavior among collegiate women: a review of research on alcohol myopia theory". Journal of American College Health, 58 (6), 523-532.

LLORCA, G., MANZANO, J.M., DÍEZ, M.A., GONZÁLEZ-TABLAS, M., BLANCO, A.L. (1995). "Alcoholismo". Cuadernos de Psiquiatría.. Madrid: Jarpyo Editores, S.A.

LÓPEZ, J.R., ANTOLÍN, N., BARCELÓ, M.V., PÉREZ, M., BALLESTEROS, A.M., Y GARCÍA, A.L. (2001).”Consumo de alcohol en los escolares de un área de salud. Hábitos y creencias". Atención Primaria; 27: 159-165

MARTÍNEZ-AZUMENDI, O. (2009). "Médicos y farmacéuticos durante la Ley Seca Americana (1920-1933)". Gaceta Médica de Bilbao; 106: 115-118.

PARADA, M., CORRAL, M., CAAMAÑO-ISORNA, F., MOTA, N., CREGO, A., RODRÍGUEZ-OLGUÍN, S. Y CADAVEIRA, F. (2011). "Definición del concepto de consumo intensivo de alcohol adolescente (binge drinking)". Adicciones, 23, 53-63.

PIKO, B. F. Y KOVÁCS, E. (2010). "Do parents and school matter? Protective factors for adolescent substance use". Addictive Behaviors, 35, 53-58.

REBOUSSIN, B., IP, E. AND WOLFSON, M. (2008). "Locally dependent latent class models with covariates: an application to under-age drinking in the USA". Journal of the Royal Statistical Society, 171, 877-897.

SCAIFE, J.C. Y DUKA, T. (2009). "Behavioural measures of frontal lobe function in a population of young social drinkers with binge drinking pattern". Pharmacology, Biochemistry and Behavior, 93, 354-36.

7.3 Publicaciones web

BABÍN, F. Y AGENCIA EFE. (2013). "El Gobierno estudia multar a los padres de menores con repetidos comas etílicos". Consultado el 14 de junio de 2013. http:// www.efe.com/efe/noticias/espana/sociedad/

FUNDACIÓN PFIZER (2012). "Juventud y Alcohol". Consultado el 28 de noviembre de 2012. http:/www.fundacionpfizer.org/docs/pdf/ 
MINISTERIO DE EDUCACIÓN (2011). "Datos y Cifras Curso escolar 2011/2012". Consultado el 18 de septiembre de 2012. http://www.educacion.gob.es/dctm/ ministerio/

PONS DIEZ, J. Y BERJANO PEIRATS, E. (1999). "El consumo abusivo de alcohol en la adolescencia: un modelo explicativo desde la Psicología social". Plan Nacional sobre Drogas. Consultado el 6 de febrero de 2012. http://www.pnsd.msc.es/ Categoria2/publica/pdf/

UNIDAD DE EVALUACIÓN DE CALIDAD. (2012) "Estadísticas de Gestión. Universidad de Salamanca". Extraído el 18 Septiembre, 2012. http://campus.usal. es/ estadisticasgenerales/index.php

\section{Las autoras:}

Ángeles Diez: Doctora por la Universidad de Salamanca. Profesora Titular de Psicología Médica. Área de Psiquiatría. Facultad de Medicina. Universidad de Salamanca.

Gloria $\mathrm{M}^{\mathrm{a}}$ Bueno: Especialista en Psiquiatría. Doctora por la Universidad de Salamanca. Profesora del Ärea de Psiquiatría. Facultad de Medicina. Universidad de Salamanca.

$\mathrm{M}^{\mathrm{a}}$ Ángeles Llorca: Doctora por la Universidad de Granada. Periodista. Licenciada en CC de la Información por la Universidad de Salamanca. Más de diez años de experiencia profesional en diversos medios de comunicación (prensa, radio, digital).

Beatriz Cabrejas: Licenciada en Psicología y Doctora por la Universidad de Salamanca. Colaboradora en la Asociación Alcohólicos Anónimos. Participación en el Programa de Estancias en las Universidades de Turku, Finlandia y Ruprecht-Kar1s-Universität Heidelberg, Alemania).

Teresa Gallego: Licenciada en Psicología por la Universidad del País Vasco y Doctora por la Universidad de Salamanca. Colaboradora en el Área de Psiquiatría. 